\title{
¿Es posible desarrollar el pensamiento crítico a través de la resolución de problemas en fissica mecánica?
}

\author{
Ignacio Laiton Poveda \\ Escuela Tecnológica. Instituto Técnico Central de Bogotá. Colombia. \\ E-mail:ilaiton@gmail.com
}

[Recibido en Junio de 2010, aceptado en Noviembre de 2010]

\begin{abstract}
Surge el presente proyecto de una reflexión sobre las características básicas de un estudiante, que lo doten para ser capaz de enfrentar adecuadamente su vida académica y cotidiana de la manera más competente posible. Se parte de la consideración de que el pensar críticamente puede constituirse en un importante aporte al desarrollo esperado del estudiante desde el punto de vista cognitivo, de donde se deduce que su enseñanza debe acometerse desde un proyecto estructurado y planeado, argumento para desarrollar una propuesta metodológica en la búsqueda de este objetivo, y que constituyó la base para el presente proyecto. En el presente artículo se pretende dar cuenta del proceso seguido, así como de los resultados finales de la investigación que sobre la enseñanza del pensamiento crítico se diseño y aplico en las aulas de la Escuela Tecnológica Instituto Técnico Central de Bogotá. Se incluye aquí la fundamentación teórica básica del trabajo en pensamiento crítico, resolución de problemas, y en el diseño de la intervención pedagógica aplicada, finalmente se muestran los resultados, ya consolidados del trabajo desarrollado durante los años 2008 y 2009, con los cursos correspondientes de Física I asignados al investigador. Se observa una tendencia clara en el comportamiento de los indicadores sobre la presencia de características de pensamiento crítico, aumentando sostenidamente a lo largo de la intervención.
\end{abstract}

Palabras claves: pensamiento crítico; autonomía; resolución de problemas; planeación.

\section{Is it possible to develop the critical thought by problems resolving in mechanical physics?}

This project arises from a reflection on the basic features of a student, it equips to be able to adequately address their academic and daily life in the most competent possible. Is intended to account for the final results of the research on the teaching of critical thinking was developed and applied in the classrooms of the Technological School Central Technical Institute in Bogota. This includes the basic theoretical foundation of work in thinking and critical thinking, problem solving, the design of applied educational intervention, and finally shows the results, and consolidated the work developed during the years 2008 and 2009, with courses Physics I allocated for the researcher. There is a clear trend in the behavior of the indicators of the presence of features of critical thinking, increasing steadily throughout the semester.

Keywords: Critical Thinking, autonomy, problem solving, planning.

\section{Introducción}

La reflexión sobre los objetivos de la educación, mas allá de la inmersión mecánica del individuo en un sistema productivo que no tiene en cuenta su individualidad debe ser el punto de partida para la actual labor docente latinoamericana. El desarrollo del pensamiento crítico, se constituye en una opción importante que debe ser considerada con seriedad y sistematicidad en la educación, la oportunidad desde las aulas de clase de construir en los estudiantes habilidades de un pensador crítico no debe echarse más en saco roto. El asunto de la enseñanza del Pensamiento crítico nos invita a releer con detenimiento lo que los autores definen con precisión debe ser un pensador crítico, la primera ventaja que se encuentra es que al interactuar con los docentes de la Escuela, la gran mayoría de ellos está de acuerdo en que le gustaría encontrar en sus aulas estudiantes críticos. Sin embargo, en la mayoría de los casos se tiende a creer, que los docentes que precedieron no lograron este cometido, pero que, en cambio, si se está logrando en nuestras clases. Es posible que para la primera afirmación se tenga algún sustento en la elaboración y aplicación que se hace de una prueba diagnóstica al 
iniciar un curso, ya sea oral o escrita, lo cual proporcionará información sobre el estado inicial de nuestros estudiantes.

En lo que concierne a la segunda afirmación, es de temer que no se tiene la suficiente autoridad ni claridad para afirmar que el método, la didáctica y la forma de la evaluación, entre otros, están alineadas de manera adecuada, tal que generen un verdadero pensamiento crítico en nuestros estudiantes. Se resalta aquí la coherencia con la que se debe diseñar un curso, considerando sus diferentes aspectos de manera integral, y no solo concentrado en el punto de los contenidos, detalle sobre lo que se suele insistir al diseñar un plan de trabajo. Entonces se debe reflexionar sobre las actividades cotidianas en el aula, preguntarse si aquellas resultan motivadoras de acciones reflexivas en el estudiante, y si permiten que sea lo suficientemente partícipe de su propio proceso formativo.

\section{Marco teórico}

\section{Primeras consideraciones}

Se parte de la afirmación de que el pensamiento crítico debe ser asumido desde la enseñanza de todas las áreas. Si se quiere que los estudiantes sean capaces de transformar la realidad cotidiana circundante, enfocados hacia el mejoramiento de condiciones sociales, sean creativos, manejen adecuadamente los problemas ambientales, etc. en contraposición a la repetición de contenidos, a que sean receptores de "saber", realizadores de tareas mecánicas de origen técnico, manipuladores de objetos o dispositivos, adaptados mansamente a la realidad, con muy pocos atisbos de creatividad en la solución de situaciones complejas y novedosas de su vida cotidiana, o en el diseño de nuevos mecanismos y dispositivos ingeniosos. Queda implícito en esto, que se parte del supuesto de que el pensamiento crítico no solo aplica para la enseñanza de la física, razón de ser del proyecto actual, sino más bien debe involucrar todas las ramas del saber, en la amplitud que dicha palabra pueda mostrar a cada escucha, y aún más en lo que se refiere a la vida cotidiana, olvidada razón de ser de la educación en las aulas, considerada desde este proyecto como importante opción de cambio social perdurable y sustentable. Se explicita entonces, que la enseñanza del pensamiento crítico no busca un engranaje mecánico del individuo en el sistema, sino que implica la generación de diferentes visiones de futuro para nuestros países, debemos actuar en consecuencia, dejando la tarea para los estudiantes de caminar como verdaderos constructores de futuro, a partir de las secuencias coyunturales de las que se conforma el presente (Zemelman, 2003).

\section{¿Qué es pensar?}

Se puede iniciar esta respuesta recurriendo a uno de los primeros autores al que se le atribuye haber hablado formalmente de pensamiento crítico, bajo la acepción de pensamiento creativo, Dewey (2007) afirma que "...a esta incontrolada corriente de ideas que pasan por nuestra mente..." en cualquier momento de nuestro día, se le da el nombre de pensamiento (Dewey, 2007, p 20), sin embargo también afirma que se puede llamar así a las cosas que no se perciben, ni se tocan, ni se huelen. Las ideas y secuencias que con absoluta coherencia se hilan al relatar una historia imaginativa, constituyen este tipo de pensar, aún así, continúa el autor que también se da el término de pensamiento, considerado como creencia, (Dewey, 2007, p22), se puede ejemplificar este tipo de pensar, al retomar afirmaciones como: los hombres pensaban que la tierra era plana, que constituyen paradigmas de pensamiento de cierta época. Constituyen, entonces, creencias que se transmiten de generación en generación o de cultura en cultura, sin un sustento verdadero o real sobre el cual dialogar o confrontar, simplemente sustentado en la tradición, la instrucción o la imitación. Se parte también, del requerimiento 
natural del estudiante como ser histórico, natural, individual pensante, asumiendo que no se puede provocar la capacidad de pensar en ninguna criatura que no piense ya espontáneamente (Dewey, 2007).

\section{Pensamiento crítico}

"La mera sucesión de ideas o sugerencias constituye el pensamiento, pero no el pensamiento reflexivo, no la observación y el pensamiento dirigidos a una conclusión aceptable" (Dewey, 2007). Se parte de esta afirmación para empezar a caracterizar al pensador crítico, tarea que han acometido diversos autores destacados como Robert Ennis, Peter Facione, Richard Paul, quienes coinciden en resaltar que el pensamiento crítico se erige como necesario en nuestro mundo actual. Incluso Boisvert (2004) señala que se destaca la importancia de facilitar a los estudiantes los medios para protegerse de manipulaciones y explotaciones, así como de vaivenes políticos. $\mathrm{Y}$ es en este sentido, que al intentar renovar el interés por la propia profesión docente, se deben generar, o al menos proponer cambios educativos en las diversas instituciones universitarias encaminados a fomentar en los estudiantes capacidades tales como "aprender a aprender" en las diversas áreas y asignaturas. Generar la inquietud en los estudiantes para que no solo adquieran un conocimiento ya elaborado que constituye la cultura y la ciencia de nuestra sociedad (Pozo, 1994), sino que también, y de manera particularmente especial, que adquieran habilidades y estrategias que les permitan aprender y evaluar por si mismos nuevos conocimientos. El estudiante, sin embargo, encuentra que la academia no se las da, de tal forma que cuando arriban a su primer semestre universitario carecen del arsenal necesario para acometer dichas tarea.

Aunque no es posible encontrar una definición unánime y uniforme de lo que se denomina "pensamiento crítico", algunas pesquisas y rastreos han llevado a encontrar cierta unidad de términos y expresiones que tienden a describir aquellas características esperadas en los sujetos con dicha forma de pensamiento, lo que facilita, en primer lugar ir interiorizándolas, pero también ir agrupándolas e identificando claramente grupos de trabajo que elaboran teorías firmes sobre el tema. Dewey (2007), define el pensamiento reflexivo como: "La consideración activa, persistente y cuidadosa de una creencia o forma supuesta de conocimiento a los luz de los fundamentos que la apoya y de las conclusiones a las que tiende". Ennis (1985) plantea que pensar críticamente es: "Un pensamiento razonado y reflexivo, orientado a una decisión de que creer o hacer". Paul (1992) plantea que "el pensamiento crítico es disciplinado y autodirigido, y ejemplifica las perfecciones del pensar adecuado ante un modo o área particular disciplinar" entendiéndolo mejor como la habilidad de los sujetos para hacerse cargo de su propio pensamiento.

\section{Resolución de Problemas}

Un segundo aspecto que inunda el presente trabajo, lo constituye la resolución de problemas, aspecto que también ha sido abordado por multiplicidad de autores, entre los que se debe destacar en primer lugar a Pozo, quien inicia su disertación afirmando, que fomenta en los alumnos la capacidad de aprender a aprender (Pozo, 1999) tan implícita en el pensamiento crítico, y que desde ya avizora el grado de complementariedad que estos dos aspectos guardan.

La resolución de problemas y el razonamiento están íntimamente ligados, como lo argumentan varios autores como Sternberg (1987), Polyá (1965), Pozo (1999), Boisvert (2004),ya que implica el desarrollo de un proceso organizado mentalmente, lo cual a su vez requiere de una elevada complejidad y profundidad en los pasos que conllevan a la respuesta, actividades mentales, conductuales y hasta afectivas, están implicadas en el proceso, enseñar a resolver problemas implica dotar a los alumnos de destrezas y estrategias eficaces, así como la creación de hábitos y actitudes para encontrar el camino a la respuesta. (Pozo, 1999). 
Sternberg (1987) reconoce que la solución de problemas ha sido caracterizada en detalle por diferentes autores y estudiada en muchas investigaciones, así como definida desde diferentes perspectivas teóricas y prácticas, así pues podemos citar a Woodworth y Schlosberg (1987, citados por Sternberg), quienes definen una situación problema cuando el individuo tiene un objetivo, pero no dispone de una vía clara para conseguirlo. Otras aproximaciones más recientes lo definen como todo aquello que asombra y desafía el espíritu hasta el punto de volver incierta la creencia (Dewey, 2007), ya que toda situación de cambio repentino lleva implícita un problema. Por otro lado Pozo acude a una definición clásica de problema como "Una situación que un individuo o un grupo quiere o necesita resolver y para la cual no dispone de un camino rápido y directo que le lleve a la solución" (Lester,1983, citado por Pozo,1999) definición adoptada en el presente trabajo.

En lo concerniente al proceso de resolución de problemas, se asume en la investigación que se presenta, el modelo presentado por Polya (1965), que retoma Pozo (1999), y que consiste en las etapas siguientes:

- Comprender el problema.

- Concebir un plan.

- Ejecución del plan.

- Visión retrospectiva.

Se debe cerrar este apartado reiterando que la solución de problemas y el pensamiento crítico son dos aspectos íntimamente ligados. Algunos autores, entre ellos Ennis (1985), enuncia dentro de las capacidades y habilidades propias del pensamiento crítico, la resolución de problemas, sin embargo otros autores como Pozo (1999), señalan la importancia de partir de una situación problémica, si queremos desarrollar pensamiento. En este sentido, nos unimos a Boisvert (2004) al recomendar que se relacione la enseñanza de las habilidades de pensamiento crítico a un marco de toma de decisiones y resolución de problemas, y que se debe estructurar la enseñanza del pensamiento crítico en torno a estrategias de resolución de problemas, como marco útil para el dominio de habilidades de pensamiento.(Boisvert, 2004).

En el marco de un curso de física mecánica para estudiantes de ingeniería de primeros semestres, se identifica como nuestro principal objetivo el desarrollo del pensamiento crítico a través de la resolución de problemas de física mecánica, diseñando nuestra intervención a partir de los postulados enunciados por Biosvert (2004), quien en este aspecto se constituye en el principal investigador, ya que realiza aportes para su implementación en un curso disciplinar, en contraposición con otros investigadores como Ennis (2000) quien considera que se debe enseñar como asignatura independiente. Es esta la razón por la que se siguen los lineamientos del autor citado en cuanto a la implementación de una intervención pedagógica.

\section{Planteamiento del problema}

Diariamente, en la labor educativa se encuentra hechos que motivan a enriquecer la quehacer docente, uno de esos varios aspectos se relaciona con lo que se desea encontrar en cada uno de los estudiantes, características que de alguna manera se asume que estén allí, como insumo básico para el trabajo; motivación, creatividad, razonamiento lógico, pensamiento deductivo, etc. Son algunos de estos elementos.

Para la presente investigación, el encontrar con frecuencia la dificultad en los estudiantes de Física, de generar respuestas agudas, críticas y creativas que muchas veces se espera de ellos, es el principal motivo. Iniciar cuanto antes el camino de solución a tal dificultad impulsó a comenzar la búsqueda de elementos que desarrollen en ellos algunos de estas características. 
Las pesquisas bibliográficas convergieron hacia lo que varios autores han denominan "pensamiento crítico", entendiéndolo como un pensamiento que proporciona razones, que reflexiona sobre las acciones, informaciones, fuentes, y que está dirigido hacia un objetivo. Se centró entonces, la investigación, en la búsqueda de estrategias que desarrollen el pensamiento crítico en los estudiantes de primeros semestres de Universidad, en particular en el área de Física Mecánica, aprovechando la resolución de problemas propio de dicha área. Se pretende dar aplicación a una propuesta en pos de superar la dificultad antes señalada. En este sentido la pregunta de investigación es: ¿la resolución de problemas de Física mecánica, desarrolla el pensamiento crítico en los estudiantes?

\section{Metodologia}

El proyecto se desarrolló con una metodología cuantitativa de acuerdo con Hernández Sampieri (2007) ya que, entre otras cosas, la aplicación sucesiva de test a lo largo de la intervención, se apoya en análisis estadísticos, su diseño coincide con el que Campbell y Stanley (1966, citado por Sampieri, 2007) denominan de series cronológicas, las variables se identificaron como: variable independiente, resolución de problemas, variable dependiente, pensamiento crítico.

En primer lugar se diseñó y aplicó en el segundo semestre del año 2008, a manera de prueba piloto, un pre-test,cuya confiabilidad se determinó a través de la Medida de estabilidad test retest al aplicar la prueba en dos momentos distintos al mismo grupo, obteniendo resultados positivos del orden del $82 \%$ al eliminar un cuarto criterio incluido en la segunda habilidad, que presentaba resultados por debajo del $10 \%$. La validez del instrumento se fundamenta en la teoría que los autores expertos en pensamiento crítico exponen y que fueron guía para la elección de las habilidades a enseñar y los criterios adecuados en la operacionalización de las variables, aspectos reseñados en el siguiente apartado.

La población en la cual se desarrolló la investigación corresponde a la totalidad de estudiantes de Física mecánica en la Escuela Tecnológica Instituto Técnico Central, que para el primer semestre de 2009 era de 5 cursos de 28 estudiantes en promedio cada uno, la muestra elegida la conforman los estudiantes del grupo E2C de Física mecánica pertenecientes a la carrera de Ingeniería Electromecánica de la Escuela Tecnológica Instituto Técnico Central de Bogotá, institución Universitaria de carácter público, 30 estudiantes de segundo semestre, a quienes se les invito a participar en el estudio durante el desarrollo del curso en cuestión, clasificada como muestreo intensionado (Sampieri, 2007 ). Ya que la metodología es cuantitativa, se desarrolla un análisis estadístico descriptivo, de medias, desviación estándar, etc. Para efectos del análisis estadístico se tuvieron en cuenta los 25 estudiantes que presentaron con regularidad las pruebas.

\section{Metodología de aula}

Para el primer semestre del año 2009, se desarrolló la aplicación formal de la intervención pedagógica, el objetivo en este apartado consiste en describir el proceso metodológico y pedagógico seguido para dicha intervención. Recordemos que, de acuerdo con el diseño de series cronológicas, se toma el pre-test para ser aplicado al comienzo de la intervención, para posteriormente generar tres momentos de situación problémica, cuyo ejemplo veremos más adelante, dentro de los cuales se intercala un test correspondiente, para finalmente proceder a la aplicación del pos test. Siguiendo a Boisvert (2004) en el aspecto pedagógico, dividimos nuestro trabajo en naturaleza, enseñanza y evaluación del pensamiento crítico. En lo relacionado con la naturaleza del pensamiento crítico y la resolución de problemas ya han sido 
mencionados con anterioridad nuestros fundamentos, enfocándonos ahora en el aspecto de la enseñanza, se adopta la siguiente secuencia:

$\mathrm{Al}$ estudiar las dimensiones, capacidades y habilidades que proponen los autores, y basado en sus criterios, en particular los enunciados por Boisvert (2004) y Ennis (2000) se decide elegir cuatro habilidades de pensamiento crítico para su enseñanza; Evaluación de la credibilidad de una fuente, análisis de argumentos, toma de postura con argumentación escrita y respeto al proceso de resolución de problemas . Es el momento para observar dichas habilidades, con sus correspondientes criterios a manera de indicadores y la forma en que se operacionalizaron, de acuerdo con la propuesta que Tamayo (2005), asignándoles un peso porcentual equilibrado ( $25 \%$ cada uno) así como los puntajes para efectos operativos y de manejo estadístico.

\section{Proceso de Generación de una propuesta pedagógica}
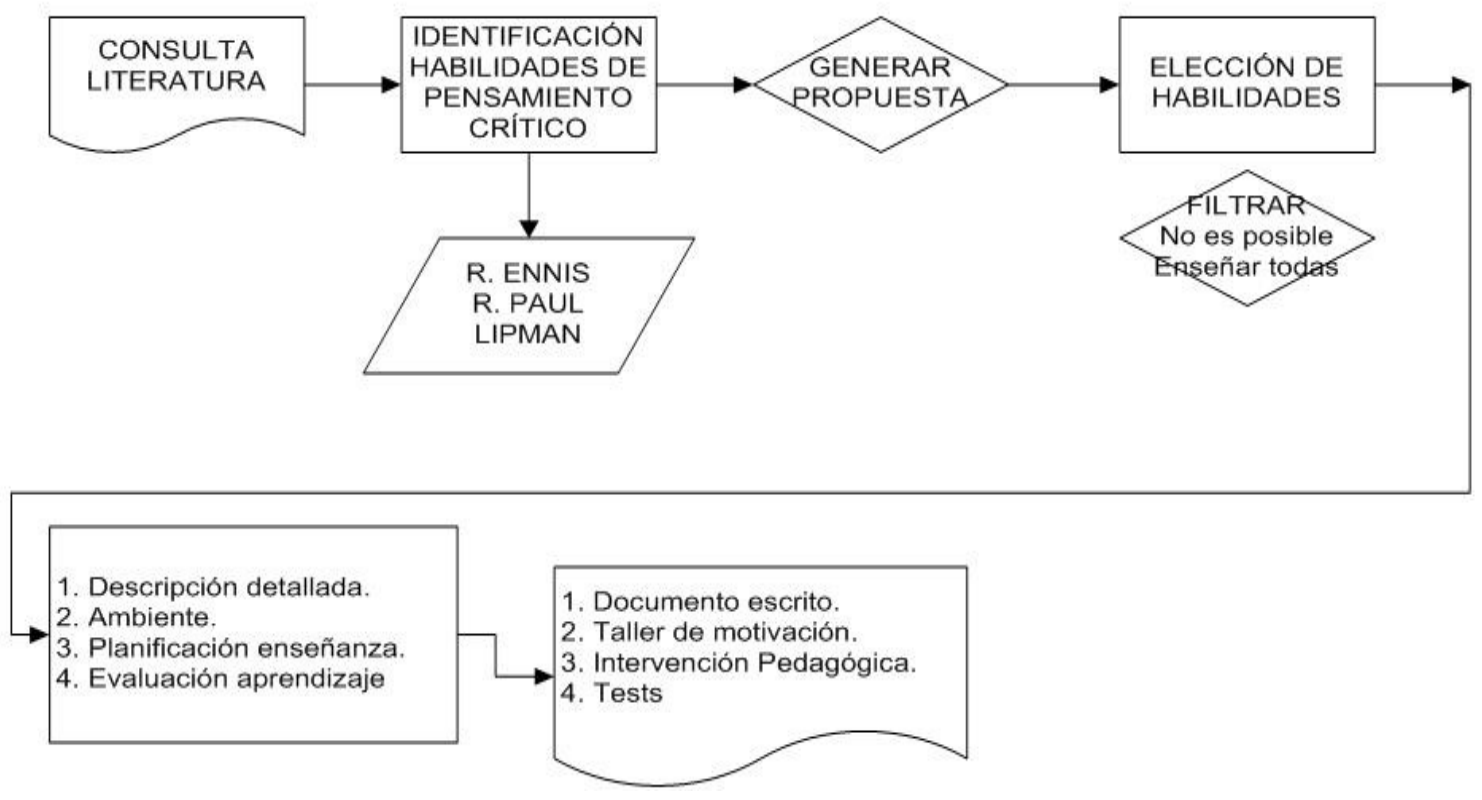

Figura 1

$\mathrm{Al}$ estudiar las dimensiones, capacidades y habilidades que proponen los autores, y basado en sus criterios, en particular los enunciados por Boisvert (2004) y Ennis (2000) se decide elegir cuatro habilidades de pensamiento crítico para su enseñanza; Evaluación de la credibilidad de una fuente, análisis de argumentos, toma de postura con argumentación escrita y respeto al proceso de resolución de problemas. Es el momento para observar dichas habilidades, con sus correspondientes criterios a manera de indicadores y la forma en que se operacionalizaron, de acuerdo con la propuesta que Tamayo (2005), asignándoles un peso porcentual equilibrado ( $25 \%$ cada uno) así como los puntajes para efectos operativos y de manejo estadístico.

Se concluye de la descripción anterior que el puntaje máximo que cada estudiante puede obtener es de seis para cada habilidad, y de veinticuatro para la totalidad de las habilidades de pensamiento crítico.

Se efectúa la descripción de las correspondientes habilidades, tomando como sustento teórico los aportes de Boisvert (2004) y Paul (2001), procediendo a la ambientación del proyecto en el aula de clase a través de un documento que explicita la metodología a usar, los aspectos a enseñar y evaluar, y motiva a los estudiantes al proceso de reflexión y discusión de cada uno 
de los aspectos contenidos en el curso. Dicha ambientación se logra subdividiéndola en dos aspectos, inicialmente se explica a los estudiantes las características del proyecto, siendo totalmente explícitos en cuanto a sus objetivos y alcances, dedicando para esto las dos primeras sesiones del semestre. En segundo término se diseña una guía para la aplicación de un taller que pretende que el estudiante identifique con claridad las habilidades que se pretenden enseñar durante el semestre y su metodología.

\begin{tabular}{|c|c|c|c|c|}
\hline \multicolumn{5}{|c|}{ HABILIDADES Y CRITERIOS ELEGIDOS EN LA INTERVENCIÓN PEDA } \\
\hline \# & HABILIDAD & CRITERIOS & ESCALAMIENTO & PUNTAJE \\
\hline \multirow{3}{*}{1} & \multirow{3}{*}{$\begin{array}{l}\text { Evaluación de la } \\
\text { credibilidad de una fuente }\end{array}$} & \multirow{3}{*}{$\begin{array}{l}\text { Experiencia, trayectoria y reputación } \\
\text { del autor, validez de la fuente } \\
\text { Nivel del lenguaje usado así como la } \\
\text { formulación matemática propuesta por } \\
\text { el autor. } \\
\text { Capacidad del autor para dar una o } \\
\text { varias razones como justificación } \\
\text { válida. Claridad en las explicaciones } \\
\text { y calidad de los ejemplos desarrollados }\end{array}$} & $\begin{array}{l}\text { El criterio aparece de } \\
\text { manera explícita y clara en } \\
\text { la respuesta }\end{array}$ & 2 \\
\hline & & & $\begin{array}{l}\text { Aparece de manera } \\
\text { tangencial y sin } \\
\text { profundidad en la } \\
\text { respuesta }\end{array}$ & 1 \\
\hline & & & $\begin{array}{l}\text { Ausencia total de } \\
\text { referencia al criterio } \\
\text { requerido }\end{array}$ & 0 \\
\hline \multirow{3}{*}{2} & \multirow{3}{*}{ Análisis de argumentos } & \multirow{3}{*}{$\begin{array}{l}\text { Reconocer conclusiones en un texto } \\
\text { Identificar las razones explicitas del } \\
\text { autor } \\
\text { Explicitar la estructura de una } \\
\text { argumentación }\end{array}$} & & 2 \\
\hline & & & & 1 \\
\hline & & & & 0 \\
\hline \multirow{3}{*}{3} & \multirow{3}{*}{$\begin{array}{l}\text { Toma de postura con } \\
\text { argumentación escrita }\end{array}$} & \multirow{3}{*}{$\begin{array}{l}\text { Emplear el vocabulario adecuado de } \\
\text { acuerdo al nivel al que pertenece. } \\
\text { Expresar de manera explícita, la } \\
\text { idea esencial de su postura o } \\
\text { presentación. } \\
\text { Ofrecer elementos que permitan } \\
\text { entender su argumentación, dando a } \\
\text { conocer razones que expliquen su } \\
\text { postura. }\end{array}$} & $\begin{array}{l}\text { El criterio aparece de } \\
\text { manera explícita y clara en } \\
\text { la respuesta }\end{array}$ & 2 \\
\hline & & & $\begin{array}{l}\text { Aparece de manera } \\
\text { tangencial y sin } \\
\text { profundidad en la } \\
\text { respuesta }\end{array}$ & 1 \\
\hline & & & $\begin{array}{l}\text { Ausencia total de } \\
\text { referencia al criterio } \\
\text { requerido }\end{array}$ & 0 \\
\hline \multirow{3}{*}{4} & \multirow{3}{*}{$\begin{array}{l}\text { Respeto al proceso de } \\
\text { resolución de problemas }\end{array}$} & \multirow{3}{*}{$\begin{array}{l}\text { Precisa con claridad los elementos del } \\
\text { problema a solucionar. } \\
\text { Formula un conjunto de soluciones o } \\
\text { parámetros tendientes a dar solución a } \\
\text { la situación. } \\
\text { Pone en práctica la mejor solución } \\
\text { antes analizada, dándole solución } \\
\text { satisfactoria a la situación. }\end{array}$} & & 2 \\
\hline & & & & 1 \\
\hline & & & & 0 \\
\hline
\end{tabular}

Tabla 1. Habilidades a enseñar, criterios y puntajes asignados.

La planeación de la enseñanza se basa en dividir el curso en tres grandes núcleos a saber:

- Cinemática.

- Dinámica.

- Trabajo y Energía.

Dentro de los cuales se programan para cada uno las siguientes actividades: 
- Elección de una situación problemica de su vida cotidiana, acorde con la temática tratada en el núcleo correspondiente, a manera de ejemplo es posible citar un trabajo elaborado por un grupo de estudiantes del grupo intervenido, quienes estudiaron el juego del tejo, juego autóctono Colombiano, declarado deporte nacional de Colombia por el congreso Nacional, que consiste en el lanzamiento de un disco metálico, con el objetivo de ubicarlo lo más cerca de posible de un bocín o aro metálico ubicado a unos trece metros del jugador, dentro de un espacio con greda de aproximadamente un metro cuadrado, en donde además se ubican varias mechas que pueden reventar al ser golpeadas por el tejo. Los estudiantes investigaron sobre la reglamentación del deporte, dimensiones de la cancha, distancia de lanzamiento, peso del tejo, etc. para elaborar algunos cálculos cinemáticos y dinámicos que tendieran a describir el lanzamiento óptimo del tejo. Este informe se entrega al final del núcleo correspondiente, ya que se va construyendo teóricamente a lo largo del desarrollo del curso.

- Desarrollo de una consulta detallada sobre las bases teóricas del tema, construyendo una matriz comparativa de los conceptos, y las diferentes maneras en que los autores las abordan. Debe incluir en su consulta cuatro fuentes de textos. Además se incluye en la matriz comparativa (Figura 2), una columna tendiente a comentar las características que el autor le imprime a su escrito: lenguaje, ejemplos, ejercicios desarrollados, y cualquier otro aspecto que el estudiante considere relevante.

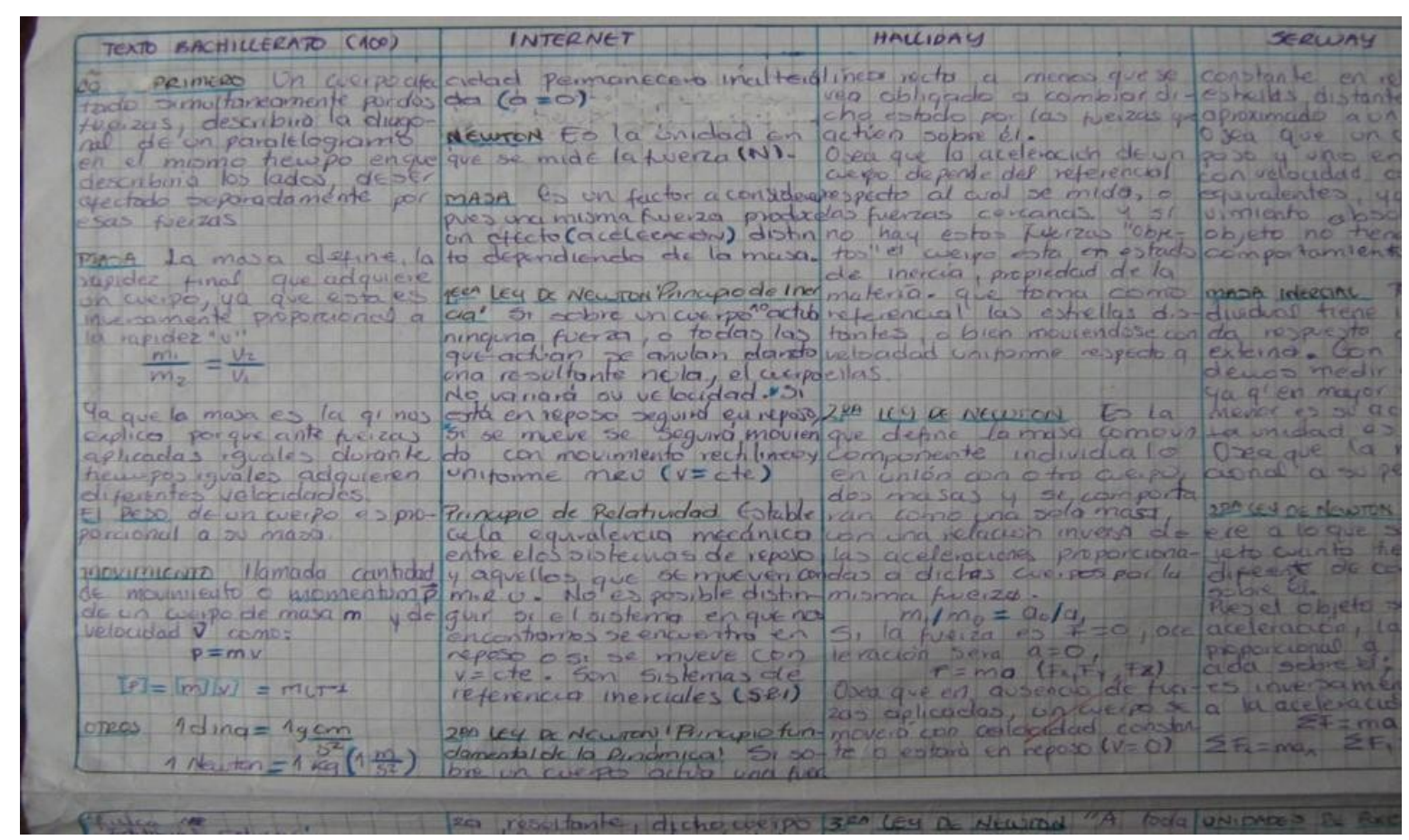

Figura 2

- En tercer término realice la lectura asignada al núcleo, sobre un tema de física relacionado o de actualidad, en el cual se debe identificar:

$\checkmark$ Argumentos o puntos de vista del autor

$\checkmark$ Identificar las razones que sustentan la argumentación del autor

$\checkmark$ Conclusiones de la lectura

$\checkmark$ Realice un diagrama que resuma el documento. 
A manera de ejemplo se puede citar el texto Alvarenga (1983) de donde se extracto la lectura "La física en los encuentros deportivos".

- Con respecto a la actividad anterior, realice un escrito en donde se exponga el punto de vista personal sobre el tema, tomando su propia posición, sustentándola con argumentos válidos. En el caso del tema de la energía, uno de los estudiantes escribió:

"mi opinión sobre el tema de la energía es que a diario estamos acabando con los recursos de la tierra para beneficio individual, sin pensar que se va a acabar algún día. Señores, estos recursos se acaban, no son infinitos ya que no se están cuidando el origen de ellos, la tierra no nos brindará más su alimento.

Debe trabajarse como dice el texto, sobre energias alternativas y renovables que no causen tanto daño al ambiente. Creo que debemos retroalimentarnos sobre el tema, conocer que se está baciendo por mejorar y tratar de dar ideas".

- Finalmente aplique el proceso de resolución de problemas a partir de las explicaciones y ejercicios dadas en clase, para solucionar la situación problemática expuesta en el primer paso, aplicándolo en la vida real, y construyendo un proyecto presentado en forma de trabajo final para cada núcleo. En este caso y retomando el ejemplo expuesto en el primer paso, los estudiantes anexaron fotos de la cancha de tejo, sobre las mediciones hechas y desarrollaron y expusieron en su trabajo todos los cálculos y pasos seguidos para la resolución de la situación. Generan sus propias preguntas, por ejemplo sobre el ángulo de lanzamiento, velocidad del mismo, etc. plantean sus requerimientos y posibilidades, identificando qué información la da el mismo escenario y qué información debe calcularse, entre otros. Es posible retomar el proceso en la gráfica de la figura 3.

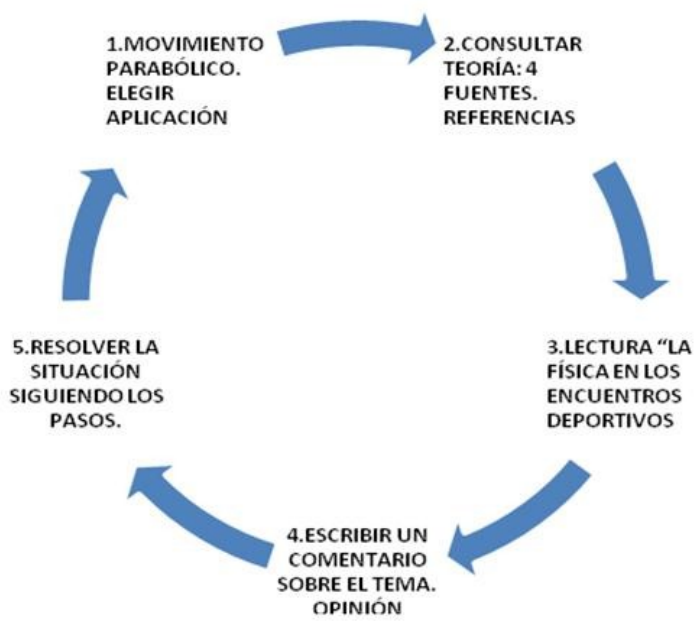

Figura 3. Ciclo pedagógico de cada núcleo.

Con el objetivo de dar cierre a este apartado, se debe mencionar que la evaluación de la calidad de la enseñanza fue medida a través del uso de los dos test durante el curso, así como con el pretest y el postest, validados a través de las revisiones y recomendaciones, así como de la aplicación de una prueba piloto antes referenciada para perfilar adecuadamente algunas preguntas. En el anexo 1 se encuentra un ejemplo de los test aplicados.

\section{Analisis estadístico, elementos generales}

Con el objeto de describir el procedimiento estadístico seguido para el tratamiento de los datos se toman elementos de cada segmento del proceso, para después realizar una mirada global a todo el proceso. 
En primer lugar se observa una tabla de organización de resultados, en este caso para el test \# 2 , recordando que se aplica uno después de cada núcleo de trabajo.

\begin{tabular}{|c|c|c|c|c|c|c|c|c|c|c|c|c|c|}
\hline \multicolumn{14}{|c|}{$\begin{array}{r}\text { TABLA DE EVALUACION HABILIDADES } \\
\text { TEST } N^{\circ} 2\end{array}$} \\
\hline \multirow[t]{2}{*}{ PERSONA } & \multicolumn{3}{|c|}{$\underset{1}{\text { HABILIDAD }}$} & \multicolumn{3}{|c|}{$\begin{array}{l}\text { HABILIDAD } \\
\quad 2\end{array}$} & \multicolumn{3}{|c|}{$\begin{array}{l}\text { HABILIDAD } \\
3\end{array}$} & \multicolumn{3}{|c|}{ HABILIDAD 4} & \multirow{2}{*}{\begin{tabular}{|l} 
TEST 2 \\
TOTAI
\end{tabular}} \\
\hline & $\mathrm{C} 1$ & $\mathrm{C} 2$ & $\mathrm{C} 3$ & $\mathrm{C} 1$ & $\mathrm{C} 2$ & $\mathrm{C} 3$ & $\mathrm{C} 1$ & $\mathrm{C} 2$ & $\mathrm{C} 3$ & $\mathrm{C} 1$ & $\mathrm{C} 2$ & $\mathrm{C} 3$ & \\
\hline 1 & 0 & 0 & 2 & 2 & 0 & 1 & 1 & 1 & 0 & 1 & 0 & 1 & 9 \\
\hline 2 & 1 & 2 & 2 & 1 & 2 & 2 & 2 & 2 & 1 & 1 & 0 & 1 & 17 \\
\hline 3 & & & & & & & & & & & & & 0 \\
\hline 4 & 0 & 1 & 1 & 1 & 0 & 0 & 1 & 2 & 2 & 1 & 1 & 0 & 10 \\
\hline 5 & 0 & 0 & 0 & 2 & 2 & 1 & 2 & 2 & 1 & 1 & 0 & 0 & 11 \\
\hline 6 & 0 & 0 & 0 & 0 & 0 & 0 & 1 & 1 & 0 & 0 & 1 & 0 & 3 \\
\hline 7 & 0 & 1 & 1 & 2 & 1 & 0 & 1 & 1 & 1 & 0 & 1 & 0 & 9 \\
\hline 8 & 0 & 2 & 1 & 0 & 0 & 0 & 1 & 1 & 0 & 0 & 1 & 0 & 6 \\
\hline 9 & 0 & 1 & 2 & 0 & 1 & 0 & 1 & 1 & 0 & 1 & 1 & 1 & 9 \\
\hline 10 & & & & & & & & & & & & & 0 \\
\hline 11 & 1 & 1 & 2 & 1 & 1 & 2 & 1 & 1 & 1 & 2 & 1 & 1 & 15 \\
\hline 12 & 0 & 0 & 1 & 0 & 1 & 0 & 1 & 1 & 0 & 0 & 0 & 0 & 4 \\
\hline 13 & 0 & 1 & 2 & 2 & 2 & 1 & 1 & 1 & 0 & 2 & 1 & 0 & 13 \\
\hline 14 & 0 & 0 & 0 & 0 & 0 & 0 & 1 & 0 & 0 & 1 & 1 & 1 & 4 \\
\hline 15 & 1 & 2 & 1 & 1 & 1 & 2 & 1 & 2 & 0 & 0 & 0 & 0 & 11 \\
\hline 16 & 1 & 1 & 1 & 1 & 2 & 0 & 0 & 0 & 0 & 0 & 0 & 0 & 6 \\
\hline 17 & & & & & & & & & & & & & 0 \\
\hline 18 & 0 & 1 & 2 & 0 & 0 & 1 & 2 & 2 & 0 & 1 & 1 & 1 & 11 \\
\hline 19 & 0 & 2 & 2 & 1 & 2 & 1 & 2 & 1 & 1 & 0 & 1 & 0 & 13 \\
\hline 20 & & & & & & & & & & & & & 0 \\
\hline 21 & 0 & 0 & 1 & 0 & 0 & 0 & 2 & 1 & 2 & 1 & 0 & 0 & 7 \\
\hline 22 & 1 & 1 & 1 & 2 & 1 & 1 & 1 & 1 & 1 & 0 & 1 & 1 & 12 \\
\hline 23 & 0 & 0 & 2 & 1 & 1 & 0 & 1 & 1 & 0 & 1 & 1 & 1 & 9 \\
\hline 24 & 0 & 2 & 2 & 1 & 0 & 0 & 1 & 1 & 1 & 2 & 2 & 1 & 13 \\
\hline 25 & & & & & & & & & & & & & 0 \\
\hline 26 & 0 & 2 & 2 & 2 & 2 & 1 & 1 & 0 & 0 & 0 & 0 & 0 & 10 \\
\hline 27 & 0 & 0 & 1 & 1 & 0 & 0 & 1 & 2 & 1 & 0 & 1 & 0 & 7 \\
\hline 28 & 0 & 0 & 1 & 2 & 1 & 0 & 1 & 1 & 1 & 1 & 0 & 0 & 8 \\
\hline 29 & 2 & 1 & 2 & 1 & 2 & 1 & 1 & 1 & 0 & 2 & 1 & 0 & 14 \\
\hline 30 & 1 & 2 & 0 & 1 & 1 & 1 & 1 & 1 & 1 & 1 & 1 & 1 & 12 \\
\hline TOTAL & 8 & 23 & 32 & 25 & 23 & 15 & 29 & 28 & 14 & 19 & 17 & 10 & 243 \\
\hline
\end{tabular}

Tabla 2. Resultados brutos test $\mathrm{N}^{\circ} 2$.

Con posterioridad a cada aplicación, se toman puntajes totales y se calculan promedios individuales y grupales, para después realizar una comparación que permita evaluar la evolución de dichos puntajes, veamos una tabla comparativa de los dos primeros test.

A partir de los puntajes y promedios, es posible construir gráficas descriptivas de la evolución de los resultados individuales, grupales y por criterio o habilidad, para finalmente globalizar promedios y puntajes de Pensamiento Crítico. Se observan a continuación gráficas que muestran algunos comportamientos relevantes. 


\begin{tabular}{|l|r|r|r|r|r|r|r|r|r|r|r|r|c|}
\hline & C1 & C2 & C3 & C1 & C2 & C3 & C1 & C2 & C3 & C1 & C2 & C3 & CURSO \\
\hline TOTAL & 7 & 12 & 6 & 19 & 17 & 16 & 24 & 24 & 16 & 11 & 24 & 9 & 185 \\
\hline Promedios & 0,3 & 0,5 & 0,2 & 0,8 & 0,7 & 0,6 & 1 & 1 & 0,6 & 0 & 1 & 0,4 & 7,4 \\
\hline
\end{tabular}

APLICACIÓN 1 TEST N 1

\begin{tabular}{|l|r|r|r|r|r|r|r|r|r|r|r|r|l|}
\hline & C1 & C2 & C3 & C1 & C2 & C3 & C1 & C2 & C3 & C1 & C2 & C3 & CURSO \\
\hline TOTAL & 8 & 23 & 32 & 25 & 23 & 15 & 29 & 28 & 14 & 19 & 17 & 10 & 243 \\
\hline promedios & 0,3 & 0,9 & 1,3 & 1 & 0,9 & 0,6 & 1,16 & 1,1 & 0,6 & 0,7 & 0,7 & 0,4 & 9,72 \\
\hline
\end{tabular}

APLICACIÓN 2 TEST N 2

Tabla 3. Puntajes totales (sobre 50) y promedios (sobre 2), obtenidos en los test $\mathrm{N}^{\circ} 1$ y $\mathrm{N}^{\circ} 2$ para cada criterio $\left(C_{\mathrm{i}}\right)$.
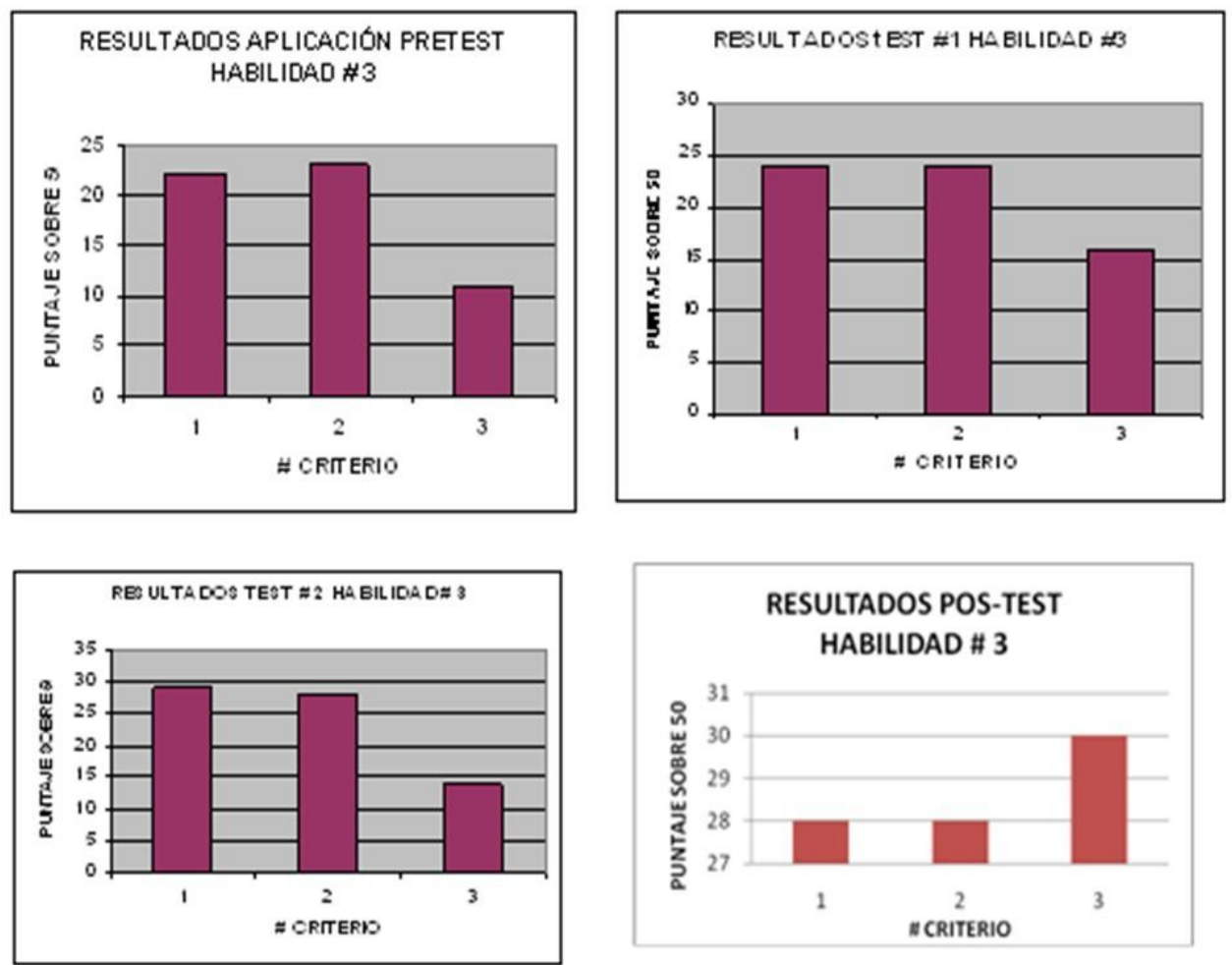

Figura 4. Evolución comparativa de los puntajes totales del curso para los criterios de la habilidad $\mathrm{N}^{\circ} 3$ a medida que se aplicaban las intervenciones pedagógicas y los test según el plan de trabajo.

A manera de ejemplo, se observa el comportamiento y evolución de los criterios correspondientes a la habilidad número 3 a través de las sucesivas aplicaciones, cuatro en total, en donde se puede observar la tendencia a aumentar el promedio de los puntajes, ya que los tres puntajes, mostraron un aumento a lo largo del proceso. Por ejemplo el criterio número dos, paso de un puntaje en el pre-test de 23 (sobre 50), a un puntaje de 28 en el pos-test. Debe notarse que se inserta esta gráfica a manera de muestra de lo que se realizó en el análisis estadístico, y que de ninguna manera es la totalidad del proceso cuantitativo realizado con los datos, proceso que resulta imposible de ser incluido en un artículo de divulgación del trabajo, y que solo se presenta de manera completa y detallada en el trabajo final de tesis de grado de la 
Maestría en Docencia de la Universidad de La Salle Bogotá, que para los interesados puede ser solicitado en su versión completa en el correo electrónico del autor.

Una segunda muestra del análisis estadístico realizado con los datos lo constituye el análisis por intervalos o clases con los puntajes totales de los estudiantes, identificando la frecuencia en cada intervalo, graficando tales resultados en porcentaje. Usando cuatro intervalos, acorde con la estadística para este rango de valores (0-24), se obtiene en la figura 5, la comparación del desempeño del grupo de estudiantes a través de la intervención pedagógica.
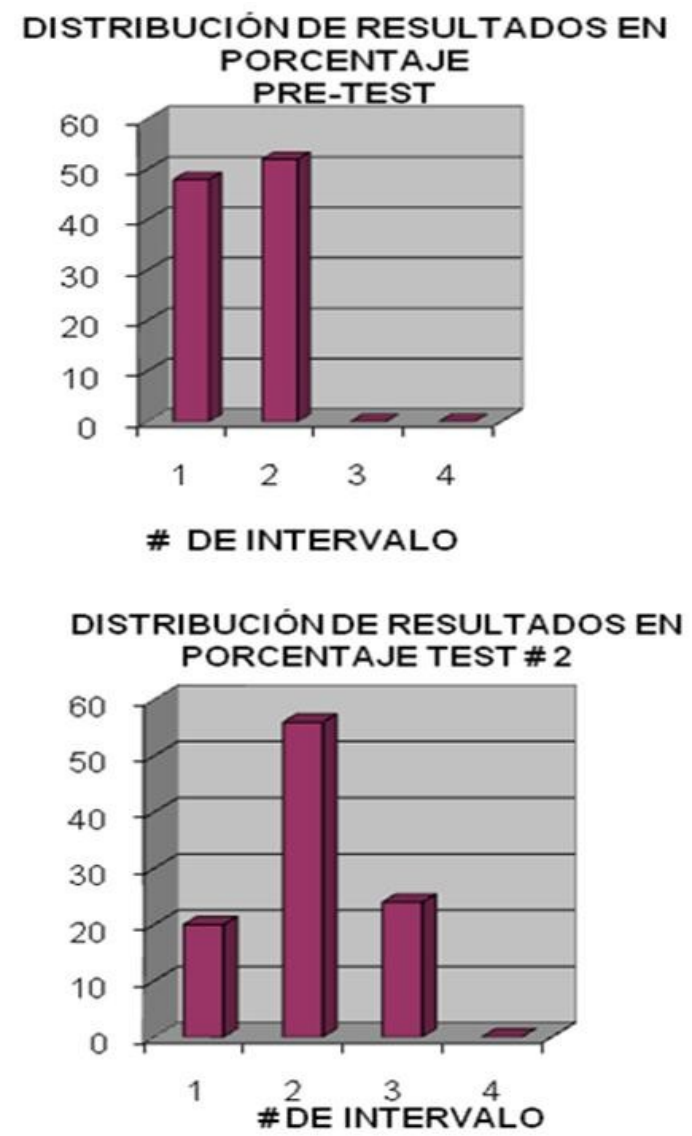

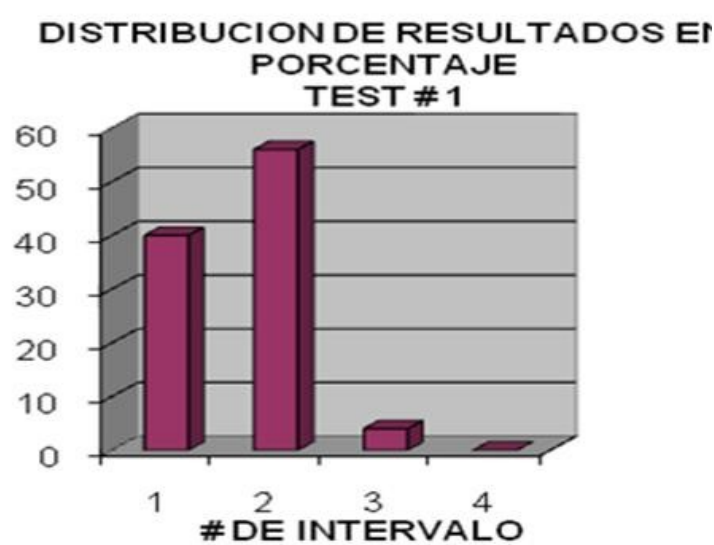

DISTRIBUCIÓNDE RESULTADOS EN PORCENTAJE POSTEST

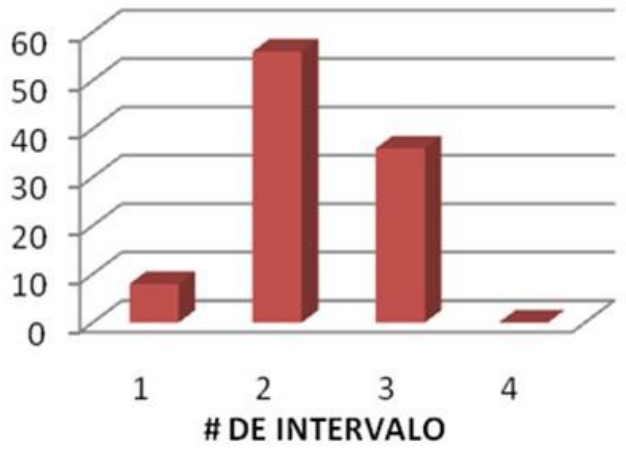

Figura 5. Distribución de puntajes totales de estudiantes para cada aplicación, por intervalos en porcentaje.

Nuevamente se observa un aumento porcentual del puntaje de los estudiantes en los intervalos dos y tres, que son puntajes entre 6 y 18 puntos sobre 24 .

\section{Resultados}

En concordancia con el enfoque investigativo y el marco teórico adoptado, se desarrolló completamente el proceso de aplicación de pre-test, intervención pedagógica y evaluación, realizando el manejo estadístico de los datos, tendiente a observar su comportamiento, aplicando estadísticos ya referenciados en el apartado anterior. Se pueden observar las siguientes tendencias, enfocado obviamente hacia la identificación de características que puedan llevarnos a aceptar o negar nuestra hipótesis de trabajo.

En el aspecto individual, se encontró como característica notoria la presencia de bajos puntajes en la generalidad de los individuos, ya que el pre-test mostro un promedio de 6.84 sobre 24, lo cual nos da una idea del estado inicial de los estudiantes en lo referente al 
pensamiento crítico y que marca desde ya el análisis que se hace de los resultados, este promedio ascendió a 11.64 en la aplicación del pos-test.

Un segundo aspecto corresponde al comportamiento de las habilidades adoptadas para la investigación, esta vez en la totalidad del grupo, en donde observamos en general una tendencia al alza a lo largo del proceso, notando, sin embargo que la habilidad de respeto al proceso de resolución de problemas es la de más difícil apropiación, ya que fue la que menos variación positiva tuvo a lo largo del proceso. Pasando de 31.33 a 39, siendo este el promedio total del curso para esta habilidad, sobre 100. Es decir que presentó un aumento del $24.48 \%$. En comparación con, por ejemplo la habilidad de análisis crítico de una fuente que paso de 22.67 a 44, aumentando en un 94\%. Es clara la tendencia de los resultados obtenidos desde esta visión.

Finalmente, al observar los puntajes totales individuales de pensamiento crítico (ver gráfica\# 4), objetivo general de nuestro trabajo, identificamos un aumento de 6,8 a 11,6 (sobre 24) desde el pre-test hasta el pos-test, esta evolución es permanente en cada uno de los test aplicados, mediados por las intervenciones pedagógicas, se presenta un aumento porcentual de 70

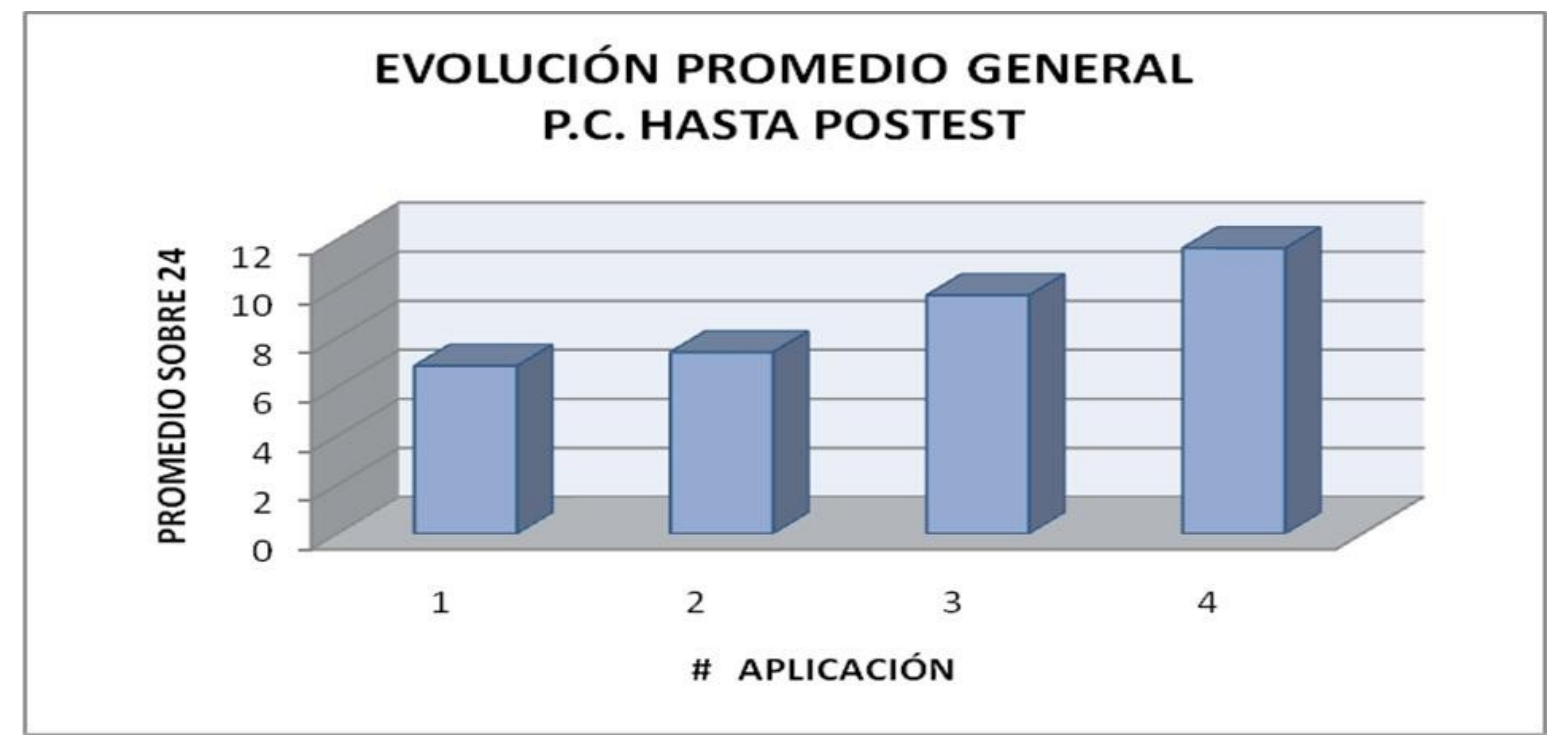

Figura 5. Comportamiento de los puntajes individuales totales desde el pre-test (1) hasta postest (4).

\section{Conclusiones}

Debe anotarse aquí que pierde sentido el leerse este apartado sino es a la luz de los anteriores resultados (apartado anterior), y de la descripción casi detallada del proceso, previamente realizada, que incluye la intervención pedagógica diseñada sobre la base de los planteamientos de Boisvert, Ennis y Pozo, y que implica los aspectos de la naturaleza, enseñanza y evaluación del pensamiento crítico a lo largo de un curso de Física mecánica. Se puede leer entonces con claridad que existe una relación entre la aplicación de la intervención pedagógica y la presencia de características de un pensador crítico en los estudiantes. En lo que respecta al objetivo general del presente trabajo, se colige que la intervención pedagógica diseñada basada en la resolución de problemas, incidió positivamente en el desarrollo de habilidades de pensamiento crítico en los estudiantes, ya que se presentó un aumento porcentual del $70 \%$ desde el pre-test hasta el post-test, a pesar de que el promedio del curso en el pos-test, que fue de 11,6, aún está muy por debajo del total de 24 posible a alcanzar. 
Se puede afirmar que los estudiantes empiezan a familiarizarse con las habilidades elegidas de pensamiento crítico, de donde a su vez se puede decir que es posible formar estudiantes más capaces de aprender a aprender, de indagar, de cuestionar, de argumentar adecuadamente, y en últimas de adquirir habilidades que les permitan desempeñarse de mejor manera en el mundo actual, que les exige cada día más poder de adaptación y aprendizaje de nuevas tecnologías, en el mundo globalizado. Se destaca nuevamente, que los anteriores criterios se aplican dentro del contexto de la enseñanza de la Física, pensándola como generadora de pensamiento crítico, dándole gran importancia a la parte conceptual de la misma, que requiere, como cualquier otro texto, de interpretación, análisis y aplicación en la resolución de problemas. Se destaca también la importancia de la autonomía que adquieren los alumnos a través del proceso, ya que son ellos los que inician cada tema al consultar cuatro fuentes, compararlas y sintetizarlas, aspecto este que mostró un gran avance porcentual a lo largo del proceso.

Finalmente se quisieran hacer algunas recomendaciones; en primer lugar resaltar la magnitud de la tarea que se puede asumir, ya que educar con pensamiento crítico a los estudiantes implica una aplicación regular en todas las áreas del conocimiento, así como en todos los niveles de la enseñanza, es natural entonces invitar al lector a ponerlo en práctica en su quehacer docente, haciendo hincapié en la necesidad de una buena planeación del curso pero bajo la premisa de que cada docente puede diseñar su propia estrategia pedagógica. En segundo lugar debemos ser humildes en reconocer que la enseñanza del pensamiento crítico implica una puesta en escena desde la educación primaria hasta la Universidad, y que en cada nivel el estudiante va adquiriendo nuevas habilidades propias de su desarrollo cognitivo y socioafectivo. Es necesario recalcar en el compromiso que los docentes tenemos con la educación de las nuevas generaciones, se propone desde este proyecto asumir el pensamiento crítico como bandera de desarrollo de la educación en Colombia, para lo cual se ha generado una propuesta tendiente a invitar inicialmente a los colegios públicos del distrito capital a que se considere el pensamiento crítico como guía para el desarrollo integral de los estudiantes.

\section{Referencias}

Boisvert, J. (2004). La formación del pensamiento crítico. Mexico. Fondo de cultura económica.

Dewey, J. (2007). ¿Cómo pensamos?, relación entre pensamiento reflexivo y proceso educativo. España. Paidós.

Heidegger, M. (2001). Conferencias y artículos. Barcelona. Ediciones del Serbal.

Pozo, J. (1994). La solución de problemas. Madrid. Santillana.

Sternberg, R. (1987). Inteligencia Humana II. Cognición, personalidad e inteligencia. Barcelona. Paidós.

Zemelman, H. (2003). Los Horizontes de la Razón. Barcelona. Antrophos. 


\section{ANEXO 1 EJEMPLO DE TEST APLICADO}

\section{TEST \# 3 DESARRDLLL DE PENSAMIENTD CRÍTICD INSTITUTO TECNICO CENTRAL ESCUELA TECNDLóGICA}

Señor estudiante:

códign:

El presente test pretende continuar el proyecto de desarrollo de pensamiento crítica implementado durante el presente semestre en la cátedra de Física l. contéstela par favar con la mayar respansabilidad y seriedad pasible.

Instrucciones:

- Usted dispone de un tiempo de 60 minutos para responderla, a partir del momento señalada por su profesor.

- Sea lo más objetiva y sincera pasible, na pretende ser una evaluación de conacimientos.

\section{× HABILIDAD \# I: ANÁLISIS CRÍTICO DE UNA FUENTE.}

En el siguiente apartado se le proporciona información sobre el tema de energía,, proporcionándose infarmación sabre sus autores, usted debe analizar y comparar las dos fuentes, emitienda un comentario sobre dicha comparación, sea la más detallada pasible. Por ejemplo, lenguaje usado, explicaciones, etc.

PRIMERA FUENTE : Willson Buffa (profesar de la Califarnia State University, Dactor en Física de la Universidad de Illinais, texto Física, quinta edición):

Conservación de la energía: la idea de fuerza conservativa nos permite extender la conservación de la energía al casa de la energía mecánica, lo cual nos es de gran ayuda para analizar muchas situaciones físicas. La suma de la energía cinética y potencial se llama energía mecánica total. En un sistema conservativo (sin fuerzas disipativas: razamiento), la energía mecánica total es canstante, es decir, se conserva: $\frac{1}{2} m v_{0}^{2}+m g h_{0}=\frac{1}{2} m v_{1}^{2}+m g h_{1}$. la anterior ecuación es la expresión matemática de la ley de conservación de la energía: "en toda sistema conservativo, las sumataria de los tipos de energía cinética y potencial es constante y es igual a la energía mecánica total del sistema".

En un sistema conservativo, las energías cinética y patencial podrían cambiar, pero sus sumatoria siempre es constante. Esta indica que las cantidades involucradas tienen una relación de balancín; si hay una disminución de la energía patencial, la energía cinética deberá aumentar en la misma cantidad para que la suma sea constante. Sin embargo, en un sistema no conservativa, generalmente se pierde energía mecánica (por ejemplo en farma de calor), en términos de la energía mecánica total tendremos que $\Delta \mathrm{E}=\mathrm{E}-\mathrm{E}_{0}$ dande $\Delta \mathrm{E}$ es la cantidad de energía que el sistema pierde. Sin embargo hay que tener en cuenta, como se señalo, que una fuerza no conservativa podría añadir energía a un sistema.

SEgunda FUENTE: (Sears , Young) (prafesar Física Carnegie University of Pittsburgh, Física Universitaria, décima edición). Suponga que un cuerpo cae libremente sin resistencia del aire, sea $v_{0}$ su rapidez en $h_{0}$ y $v_{1}$ su rapidez en $h_{1}$. el teorema del trabaja y la energía cinética dice que el trabaja total efectuado sobre el cuerpo es igual al cambio en su energía cinética: $W=E_{k}$.- $E_{D}$, pera si la gravedad es la única fuerza que actúa, tenemas que el trabajo será $W_{t}=m g h_{1}-m g h_{0}$. si juntamas estas das cosas tenemos que : $E_{k_{0}}-E_{0}=$ mgh $h_{1}-m_{g} h_{0}$ o sea que $E_{0}+m g h_{\square}=E_{1}+m g h_{1}$. ahora, si definimos la cantidad $E$ + mgh como la energía mecánica total del sistema, entendienda por sistema el formada por el cuerpo de masa m y la tierra, entonces la cantidad $E_{\square}+m g h_{\square}$ es la energía mecánica total del cuerpo en el primer mamenta $(\mathrm{t}=\mathrm{D})$ y $\mathrm{E}_{1}+$ mgh, será la energía mecánica total del cuerpo en el segunda momento. Entonces cancluimos de una de las ecuaciones que $\mathrm{E}$ total en cero es igual a E tatal en uno. Es decir E es constante, tiene el mismo valor en $h_{0}$ que en $h_{1}$. pero como las pasiciones $h_{1} y h_{2}$ san arbitrarias en el mavimiento del cuerpo, la energía total E tiene que tener el mismo valor en todas los puntos durante su mavimiento. Una cantidad que siempre tiene el mismo valor se dice que es una cantidad conservada. Si solo la fuerza de gravedad efectúa trabajo, la energía mecánica total es constante, es decir se conserva. Este es un ejempla de la que se denamina el principio de conservación de la energía. Cuanda lanzamos una pelata al aire, su rapidez al subir disminuye conforme la energía cinética se convierte en energía potencial. Al bajar, la energía patencial se convierte en cinética y la rapidez de la bola aumenta, pero la energía mecánica total es la misma en todas los putos del movimiento. 


\section{ø. HABILIDAD \# 2: ANÁLISIS DE ARGUMENTOS:}

Lea detenidamente el siguiente artículo y resuelva luega las tres actividades propuestas:

“podemos hacer una distinción general entre los sistemas, considerando dos categorías de fuerzas que podrían actuar en su interior: fuerzas conservativas y no conservativas. Ya se han visto dos fuerzas conservativas, la gravedad y la fuerza de un resarte. También se dijo de una fuerza no conservativa, la fricción. Definimos "una fuerza es conservativa si el trabajo efectuada por ella o contra ella para mover un objeto es independiente de la trayectoria del abjeto". Lo que implica esta definición es que el trabajo efectuado por una fuerza conservativa depende únicamente de las posiciones inicial y final del objeto.

En un principio puede ser difíil captar el concepto de fuerzas conservativas y no conservativas, examinaremos algunos ejemplos que nos ayuden a entenderlo. En primer lugar ¿qué significa que sea independiente de la trayectoria? Por ejemplo, si queremos subir una caja hasta una determinada altura, debemos efectuar un trabajo en contra de la fuerza conservativa de la gravedad, el trabajo efectuado para subir la caja no depende de la trayectoria de la caja, sina únicamente de las posiciones iniciales y final de la caja. la magnitud del trabajo efectuado es igual al cambio en la energía potencial y, de hecho, la energía potencial esta asaciado al concepto de fuerzas conservativas. En cambio en la energía potencial, puede definirse en términos del trabajo efectuado por una fuerza conservativa. Por otro lado, una fuerza no conservativa si depende de la trayectoria.

La fricción es una fuerza conservativa. Por ejemplo, si queremos subir la caja del ejemplo anterior, a través de una rampa con fricción, se necesitaría más trabajo, contra le fricción, entre más larga sea la rampa, más trabajo adicional se necesitaría. Es obvio entances que el trabajo total efectuado no es igual al cambio e I energía potencial del objeto, una parte del trabajo se efectúo contra una fuerza no conservativa, además de contra la fuerza conservativa de la gravedad. En este casa, la energía asociada al trabajo contra la fuerza de friccín, se convertiría en energía calorifica. Así, una fuerza conservativa permitiría conservar toda la energía en farma de energía potencial, mientras que una fuerza no conservativa no. Si moviéramos la caja sobre una mesa, solo efectuamos trabaja contra la fuerza no conservativa de la fricción, es evidente que el trabajo dependerá de la trayectoria, el trabajo sería mayor si moviesemos la caja por las orillas de la mesa hasta llegar al destino, que si la moviéramos en línea recta.

Fragmenta de Física de Buffa.

1. identifique y enuncie claramente los argumentas principales del texto:

2. identifique y enuncie la conclusión a conclusiones del texta:

3. explicite en un cuadra, resumen, mapa conceptual o tabla (o cualquier atro recurso) la estructura del texta, relacionándalo de manera clara. Por ejemplo:

\begin{tabular}{|l|l|l|}
\hline SITUACIÍN & ARGLMENTACIÍN & CLNCLUSIÍN \\
\hline & & \\
& & \\
\hline
\end{tabular}

\section{¥ HABILIDAD \# 3: TDMA DE PDSTURA CDN ARGUMENTACIDN ESCRITA:}

Se pretende realizar un evento internacional sobre el tema de energía, en la promación de mismo se lee: ".....Durante el evento se va a presentar una prospectiva de la situación energética del país en lo relacionado a disponibilidad de recursos de origen fósil en petróleo, carbón y gas, relacionando el alcance de las reservas con la intensidad de la demanda y la necesidad de trabajar en la sustitución en forma rápida de estas energéticos por energéticos renovables y amigables del medio ambiente; también se analizará la situación estratégica de Colombia en el concierto suramericano y los efectos que este consumo energético produce sobre la capa de ozono, los vientas, los fenómenos del niño y la niña, las personas y los animales" ¿qué opina usted sobre el tema de las energías? Tome una posición y arguméntela adecuadamente. 


\section{^ HABILIDAD \# 4: RESPETD AL PROCESD DE RESDLUCIÍN DE PRDBLEMAS:}

Tenienda en cuenta los pasas en las resolución de un problema, Ĺcómo resolvería usted los siguientes cuestionamientos?

Usted tiene una pelata de tenis, y esta situado en el balcán del tercer pisa del ITC, usted decide soltar la pelata desde allí. ¿Ćámo determinaría la energía patencial de la pelata en el instante en que la deja caer? ¿Puede determinar la energía cinética que pasee la pelata justo cuanda choca con el pisa? ¿Puede calcular la velocidad de la pelota en ese momenta? Nota: no es necesario realizar ningún cálcula matemática, lo puede explicar en palabras.

DOCENTE INVESTIGADOR: IGNACIO LAITON PDVEDA 